\title{
Dry Needling in Physical Therapy Practice: Adverse Events Vanessa R. Valdes
}

Mount Sinai Physical Therapy, Eleven Madison Ave., 1B, New York, USA

\section{Abstract}

Dry needling is an increasingly utilized modality among physical therapists for the treatment of musculoskeletal pain and dysfunction. Continuing educational programs targeted to licensed physical therapists have proliferated in the past decade and research into the efficacy of this intervention is accelerating. When utilizing any new treatment technique, the inherent risks involved must be weighed against the potential therapeutic benefits. Knowledge of the adverse events associated with dry needling is essential in the risk management of physical therapy practice. Understanding the frequency and types of adverse events can help minimize their occurrence.

\section{Introduction}

Dry needling (DN) has become an increasingly used modality in the treatment of musculoskeletal pain and dysfunction by physical therapists (PTs) in the USA. In 2012 the American Physical Therapy Association (APTA) updated the professional scope of practice to include DN as a skilled intervention useful in alleviating physical impairment and functional limitations [1]. Post-graduate training programs and research into the use and effectiveness of $\mathrm{DN}$ in clinical practice continues to proliferate. These educational programs are often of a short duration as they build upon the knowledge and skills already obtained in the entry-level formal training of physical therapists [2]. Internationally, dry needling courses developed for physical therapists are also of a relatively short duration and it has been concluded that the technique is easy for PTs to safely learn [3].

In some states, the addition of DN into the physical therapy scope of practice has involved protracted legal battles from the acupuncture community, which, for the most part, opposes this development. Acupuncturists claim that PTs are practicing acupuncture by performing dry needling interventions and are unqualified to do so. Due to the relatively short duration of dry needling courses, they feel that public safety is threatened [4,5]. Despite this rhetoric from the acupuncture community, there is no evidence thus far of serious adverse events associated with DN by physical therapists [4].

Both acupuncture and DN are generally considered to be low-risk interventions but serious complications, although rare, have been reported in the literature. As an invasive intervention, practitioners must be cognizant of the risks involved with this modality. The focus of this paper will be on reviewing the frequency and types of adverse events associated with $\mathrm{DN}$ and on a discussion of their prevention and treatment.

\section{What are Adverse Events?}

An adverse event (AE) is "an unexpected and undesired incident directly associated with the care or services provided to the patient; an incident that occurs during the process of providing health care and results in patient injury or death; or an adverse outcome for a patient, including an injury or complication." Unfortunately, any treatment that can have an effect can also have an adverse effect [5]. Traditionally, orthopaedic physical therapy is considered to be safe, in part because the training of physical therapists embraces traditions of wide error margins to help minimize risk [5]. Safety must be a vital consideration for physical therapists who embrace intervention modalities that have

\section{Publication History:}

Received: September 19, 2019

Accepted: October 30, 2019

Published: November 01, 2019

Keywords:

Acupuncture, Pneumothorax, Trigger point the potential for serious risks associated with them (i.e. high velocity cervical manipulation or DN).

\section{Frequency of Adverse Events Associated with Needling Modalities}

\section{A literature review}

Any invasive technique that involves a needle insertion involves risks. Broadly speaking, $\mathrm{DN}$ is an intervention modality that uses thin, solid filament needles to create a therapeutic effect when the skin is punctured. Dry needling is an umbrella term that encompasses many types of approaches (Table 1).

Currently there are no national or international systems for tracking data on AEs associated with DN or acupuncture. The usual method of tracking AEs is to conduct prospective surveys sent to either practitioners or patients designed specifically for this purpose. Selfreporting by practitioners regarding AEs is limited in both accuracy and reliability as recall and selection bias in reporting can occur. AEs may become obvious after a treatment session and a practitioner may not even be aware of their development. Frequency of AEs is therefore difficult to determine and may be underreported in prospective surveys.

Publishing case reports can also be helpful to learn about types and treatments of AEs but they do not give reliable data on frequency. Clinicians may be reluctant to admit they made a clinical error and publish a case study about it. Often the person who writes up a case study involving an $\mathrm{AE}$ is the person who ended up treating the patient after the injury had occurred (e.g., A practitioner treating a patient who sustained a pneumothorax or required therapy associated with an injury caused by a broken needle) $[5,6]$.

Adverse events may be classified according to severity: mild or minor, significant and serious [7]. Generally speaking, mild AEs are of

*Corresponding Author: Dr. Vanessa R. Valdes, Mount Sinai Physical Therapy, Eleven Madison Ave., 1B, NY 10010, USA, Tel: 212-538-7770, Fax: 212-538-7755; E-mail: vanessarvaldes@gmail.com

Citation: Valdes VR (2019) Dry Needling in Physical Therapy Practice: Adverse Events. Int J Phys Ther Rehab 5: 157. doi: https://doi.org/10.15344/2455$7498 / 2019 / 157$

Copyright: (C) 2019 Valdes. This is an open-access article distributed under the terms of the Creative Commons Attribution License, which permits unrestricted use, distribution, and reproduction in any medium, provided the original author and source are credited. 
a short duration, reversible and cause minimal inconvenience to the patient. Significant AEs will require some medical intervention and/or will interfere with a patient's activities. AEs are considered to be serious if they require a hospitalization or prolong an existing hospitalization, cause a persistent or significant disability, or are life-threatening [7]. The time frame for AE severity has not yet been clearly defined but usually mild AEs will last a few hours while significant and serious AEs can persist for days or weeks. The lack of standardization of AEs has contributed to an absence of clarity on this subject. The ability to grade AEs associated with DN (both in frequency and severity) would be helpful in developing an informed consent for patients undergoing these interventions [7].

A summary of published research from the past two decades of AEs related to acupuncture and dry needling by various practitioners is found in Table 2. Most research has focused on AEs related to acupuncture and not dry needling. Although both use the same tools (fine filament needles of varying lengths and gauges), there are differences in intervention techniques. Dry needling often involves a deeper needle insertion and needle manipulation to obtain a reaction called a local twitch response. Needle retention may not occur. Acupuncture involves needling to the depth of the acupuncture point and needle manipulation to get "de qi" $[7,8]$. Needle retention is common and may last 15-20 minutes or longer. Because of the differences in needle techniques, it is uncertain whether data based primarily on acupuncture interventions applies to DN interventions [7].

Reviews of the research in Table 2 show that design limitations and format variations limit the comparison of results. Some research analyzed practitioner-reported data while others relied on patient's responses to questionnaires. Methodology variables and lack of reliability and validity in the research limit their usefulness in determining frequency of AEs. When performed by adequately trained practitioners, both acupuncture and DN appear very safe but there have been documented fatalities and serious effects like pneumothorax and infections as a result of interventions $[7,8]$.

Only the Brady study [17] has specifically focused on AEs occurring as a result of DN performed by physical therapists. This prospective study recruited 51 volunteers from a group of 183 Irish physiotherapists who had undergone the 64-hour David G. Simons Academy trigger point DN training. The participants completed two questionnaires surveying the number of trigger point DN interventions they performed and any AEs that occurred as a result of the interventions. Bleeding and bruising were the most common AEs but all were deemed to be mild and not significant. Compared with research that looked at AEs related to acupuncture, this rate was higher

\begin{tabular}{|l|l|}
\hline Dry Needling Style & Definitation/Source \\
\hline Trigger Point Dry Needling & Myofascial trigger point model [14] \\
\hline Intramuscular manual therapy & American term associated with the Myofascial trigger point model [1] \\
\hline Intramuscular stimulation & Chan Gunn's "radiculopathy model," a neurosegmental model [67] \\
\hline Superficial Dry Needling & Baldry model [68] \\
\hline Spinal Segmental Sensitization Model & Dr. Andrew Fisher: combines features of trigger point model and Gunn's radiculopathy model [69] \\
\hline Classical or traditional acupuncture & $\begin{array}{l}\text { Acupuncture is an ancient form of Chinese medicine involving the insertion of solid filiform needles into } \\
\text { the skin at specific point on the body to achieve a therapeutic effect [70]. }\end{array}$ \\
\hline Western Medical acupuncture & $\begin{array}{l}\text { A therapeutic modality that is an adaptation of Chinese acupuncture using current knowledge of anatomy, } \\
\text { physiology, pathology and the principles of evidence-based medicine [71]. }\end{array}$ \\
\hline
\end{tabular}

(Adapted from "The Safe Practice of Dry Needling in Alberta: Summary Report" October 2014)

\begin{tabular}{|c|c|c|c|}
\hline Author of Study & Study location and type & Practitioner type & Source of results \\
\hline White et al. 2001 [72] & UK/Prospective study & Physicians, physical therapists & Practitioner \\
\hline MacPherson et al. [12] & UK/Prospective study & Acupuncturists & Practitioner \\
\hline Ernst E et al. 2001 [73] & Europe and Far East/Prospective study review & Medical physicians, acupuncturists & Practitioner or patient \\
\hline Witt et al. 2001 [20] & Germany/Prospective study & Medical physicians & Patients \\
\hline Lao L et al. 2003 [38] & UK/Review of Case reports 1965-1999 & $\begin{array}{l}\text { All included (physician, chiropractors, } \\
\text { acupuncturists, osteopaths) }\end{array}$ & Published case reports \\
\hline Melchart et al. 2004 [74] & Germany/Prospective study & Medical physicians & Practitioner \\
\hline White et al. 2006 [13] & UK/Prospective study & $\begin{array}{l}\text { Medical physicians/physical therapists/ } \\
\text { acupuncturists }\end{array}$ & Practitioners \\
\hline Ernst E et al. 2011 [75] & $\begin{array}{l}\text { International/Systematic review of clinical } \\
\text { research and case reports between 2000-2009 }\end{array}$ & Acupuncturists & $\begin{array}{l}\text { Published systematic } \\
\text { reviews and case research }\end{array}$ \\
\hline Xu et al. 2013 [48] & $\begin{array}{l}\text { International/Review of Case Reports 2000- } \\
2011\end{array}$ & $\begin{array}{l}\text { Acupuncturists/physical therapists/ } \\
\text { physicians/ Unspecified }\end{array}$ & Published case reports \\
\hline Brady et al. 2014 [17] & Ireland & Physical Therapists & Practitioners \\
\hline $\begin{array}{l}\text { McDowell JM et al. } 2014 \\
\text { [23] }\end{array}$ & $\begin{array}{l}\text { New Zealand/Cross-sectional descriptive } \\
\text { study }\end{array}$ & Physical Therapists & Practitioners \\
\hline
\end{tabular}

Table 2: Research of Adverse Events in Acupuncture and Dry Needling (2001-2018). 
but other research looked at AEs from a patient's perspective [7]. It is noted that patients often underreport an $\mathrm{AE}$ as a result of an intervention if there is no change or decline in functional status [7]. A major limitation of the Brady paper is that the data was self-reported and practitioners volunteered to participate, which may result in an inaccurate or underreporting of AE. The study has also been criticized for not reaching a target level of interventions that would be required to identify a serious AE [7].

The New Zealand Physiotherapy professional organization (PNZ) has implemented a voluntary system to report AEs in PT interventions, including dry needling and acupuncture [7]. The McDowell study reviewed 176 voluntary self-reported AEs associated with interventions performed by New Zealand physiotherapistacupuncturists from 1998 to 2013. Eighty-one percent of the AEs reported were considered to be minor. It is noted that underreporting of AEs is probable and the needling "style" could not always be determined from the volunteered reporting which could have skewed the data and conclusions.

To summarize the available data: AEs from both DN and acupuncture interventions are usually mild and transient in nature. The most common mild AEs include bruising and bleeding, pain (during and after intervention), and syncope/vasovagal response [7,8]. Significant or severe AEs are rare, although individual case studies have described injuries associated with DN. Serious AEs are commonly associated with practitioner negligence and poor adherence to practice standards [7]. These more serious AEs can include the following: pneumothorax/haemothorax, infections, serious bleeding, cardiac tamponade, nerve injuries, and broken or forgotten needles. A review of these various AEs and their prevention and intervention will follow. Precautions related to treating pregnant women will also be examined (Table 3 ).

\section{Mild Adverse Events}

\section{Bleeding and Bruising}

Mild bleeding and bruising are the most common AEs associated with DN and are difficult to prevent [7]. Bleeding can occur outwardly or inwardly as a result of needling, and small hematomas can develop in the muscles or skin. Mild pressure over the needle site with a cotton ball is usually sufficient to minimize bleeding and ensure homeostasis.

Mild bleeding and bruising are the most common AEs associated with DN and are difficult to prevent [7]. Bleeding can occur outwardly or inwardly as a result of needling, and small hematomas can develop in the muscles or skin. Mild pressure over the needle site with a cotton ball is usually sufficient to minimize bleeding and ensure homeostasis.

Screening patients for bleeding disorders (like severe thrombocytopenia), the use of anticoagulants and certain medications and supplements is recommended prior to DN interventions [7]. $\mathrm{McCulloch}$ et al. conducted a systematic review of the literature to evaluate the safety of acupuncture in patients taking anticoagulant medications [7]. The study asked the question "Does acupuncture in anticoagulated patients present a higher-than-expected risk of bleeding?" The bleeding complication rate was $1.4 \%$ (56 incidents in 3,974 intervention sessions) with the vast majority being either an asymptomatic bruise or minor drop of blood as seen in many needling procedures. None of the bleeding was considered significant. The authors concluded that acupuncture has a high degree of safety with patients taking anticoagulant medications. It is assumed that this conclusion may apply to dry needling as well.

Some practitioners advocate the use of anti-bruising topical products to help minimize bruising. Arnica Montana is a perennial herb thought to be anti-ecchymosis as a result of a vasodilation effect and possible up-regulation of macrophage activities. Despite this recommendation, the majority of randomized controlled trials show that homeopathic Arnica Montana is no better than a placebo in treating bruising, swelling and pain [7].

More serious bleeding occurs if arteries or veins are punctured during DN. Arterial bleeding is rare but will spread quickly and pulsate. Needling into blood vessels can be avoided with an understanding of underlying anatomy and palpation of the area prior to needling. If a blood vessel is punctured, a sharp, painful sensation may be felt (more commonly with arteries). Bleeding into a fascial compartment is dangerous as increased pressure can cause tissue damage. The knowledge of vascular anatomy and the avoidance of large blood vessels are essential when needling.

Case studies describing serious AEs associated with bleeding illustrate the importance of anatomical knowledge. Peuker and Gronemeyer described four cases of blood vessel lesions (including the development of an anterior compartment syndrome) as a result of acupuncture [7]. More recently, Berrigan et al. reported on the development of an epidural hematoma as a result of DN [7]. An otherwise healthy 62-year-old woman with a body mass

\begin{tabular}{|l|l|l|l|}
\hline Severity & Mild/Minor & Significant & Serious \\
\hline Frequency & Common: (1-10\%) & Uncommon: $(0.1 \%-1 \%)$ & Rare: $(0.01-.1 \%)$ \\
\hline & $\bullet$ Bleeding & $\bullet$ Prolonged pain & $\bullet$ Pneumothorax/Haemothorax \\
\hline & $\bullet$ Bruising & $\bullet$ Excessive bleeding or bruising & $\bullet$ Other organ puncture \\
\hline & $\bullet$ Pain - during/after & $\bullet$ Nerve injury & $\bullet$ Infection \\
\hline & $\bullet$ Dizziness & $\bullet$ Headache & $\bullet$ Broken needle \\
\hline & $\bullet$ Temporary symptoms aggravation & $\bullet$ Vomiting & $\bullet$ Cardiac Tamponade \\
\hline & $\bullet$ Nausea & $\bullet$ Forgotten needles & \\
\hline & $\bullet$ Sweating & $\bullet$ Seizures & \\
\hline & $\bullet$ Fatigue & $\bullet$ Severe Emotional reactions & \\
\hline
\end{tabular}

Data derived from MacPherson et al. (2001) [12], White A (2006) [13], Witt et al. (2009) [20], Brady et al. (2014) [17]. 
index (BMI) of 19.2 was treated for myofascial neck pain and had $\mathrm{DN}$ of the infraspinatus, rhomboids, trapezius and upper cervical multifidus muscles. On the $4^{\text {th }}$ treatment session, she felt a "warm sensation" throughout her body after a needle insertion. Later that day, she began experiencing increased pain and neck stiffness. Three days post-intervention she developed an altered mental status. An emergency room evaluation revealed a complex epidural hematoma, which necessitated admission into an intensive care unit. A gradual resolution of neuropathic pain symptoms occurred in the two months after hospital discharge. This case illustrates the importance of specific anatomy and needle trajectory knowledge and understanding of the relationship of needle depth and BMI.

\section{Pain during or after intervention}

Increased pain during treatments is more commonly associated with DN when compared to acupuncture. Brady et al. [17] found the frequency of pain during DN interventions to be $3.01 \%$ while the frequency of pain after the DN intervention was slightly lower $(2.19 \%)$. The pain may be the result of neuromuscular injury or hemorrhagic and inflammatory changes caused by the needling. The area of pain is often in the area that was needled or in the trigger point referral pain pattern. The number of needle insertions and the pain perceived during the needling had a positive correlation to the pain experienced post-intervention. No relationship was found between the number of local twitch responses elicited during treatment and post-needling soreness [7].

Post-intervention pain is often described as a dull aching or a constant pressure and usually will persist for less than 72 hours. Travell and Simons recommended avoidance of strenuous activities for 2-3 days post-trigger point injection (which involved a larger gauge needle) to avoid soreness but no research support this advice [7]. Patients with a low baseline of myofascial pain may show a reduced tolerance to post-treatment soreness compared with those who present with higher myofascial trigger point pain levels preintervention. Communication with patients about the possibility of some short-term post-intervention pain is recommended and may even be reframed as a positive therapeutic sign [7]. Additional therapies may help decrease post-needling soreness and include low load exercises, ultrasound, hot packs, transcutaneous nerve stimulation and kinesio tape application but minimal clinical guidelines regarding their efficacy are available.

\section{Vasovagal syncope response}

Most AEs associated with DN are related to the direct placement of a needle in the body. A vasovagal syncope response (VVR) is an autonomic nervous response that occurs as an indirect result of needling or other stimuli. The autonomic nervous system responds through sensory impulses along autonomic afferent fibers. This stimulation causes bradycardia, hypotension and syncope as a result of a transient withdrawal of sympathetic tone and an increase in vagal tone. The signs and symptoms are variable and may include dizziness, nausea, sweating and paleness. Patient may also report feeling lightheaded, developing blurred or tunneling of vision, or yawning. Symptoms can progress to eventual unconsciousness.

Vasovagal responses are a relatively rare $\mathrm{AE}$ associated with acupuncture, occurring in .02-7\% of all interventions [7]. Christensen et al. reviewed clinic data from an 18-month period of patients receiving acupuncture interventions at the University of Minnesota
Masonic Children's Hospital in conjunction with the Northwestern Health Sciences University. A retrospective chart review of 281 interventions showed 5 cases of documented VVR that resulted in intervention termination or alteration. Of these 5 cases, three were acupuncture first-timers. Common features of patients who had a VVR included those with increased stress levels, irregular sleep, and disruption of regular lifestyle patterns.

Patients and practitioners can find such events to be anxiety producing despite the quick recovery that generally occurs. Practitioners must be well versed in the prevention and treatment of VVR. Awareness of patients who have a fear of needles or phobias concerning blood or injury is essential prior to DN. Vasovagal responses are more common among patients who are nervous, fatigued, having DN for the first time, or if the patient is hungry or dehydrated [7]. Recommendations to eat and drink 1-2 hours prior to interventions are helpful. Patients should be treated in a comfortable position in a supine or prone position or a zero-gravity chair. Strong needling in a seated position may cause a marked vasodilation and lead to hypotension [7]. Minimizing the number of needles and avoiding aggressive needling stimulation dependent on patient response is important, especially in the initial treatments.

The treatment environment should be comfortable and quiet. Symptom recognition is important, and communication (both verbal and non-verbal) with patients during $\mathrm{DN}$ is essential. If a person is showing signs of a VVR, removal of the needles and elevation of the feet with the patient being supine is helpful. Monitoring of airway, blood pressure, and pulse is required. Patients who have this type of reaction should notify healthcare professionals prior to future blood draws or DN sessions.

\section{Serious and Severe Adverse Events}

\section{Pneumothorax/Haemothorax}

The AE related to DN that garners the most media attention is the development of a pneumothorax or haemothorax. It is the most common serious complication of acupuncture or DN interventions and is of legitimate concern as needling around the rib cage and upper trapezius region are common. Most patients who sustain a pneumothorax will fully recover and mortality is rare but prevention of this $\mathrm{AE}$ is essential as it has the potential to be life threatening. Physical therapists must be conversant in the clinical presentation of a pneumothorax in order to help in medical management of this condition.

A pneumothorax is the presence of air or gas (or blood in the case of a haemothorax) in the pleural cavity. A pneumothorax can be spontaneous or traumatic in nature. A spontaneous pneumothorax can be primary or secondary dependent on a concurrent pre-existing lung condition. A primary spontaneous pneumothorax is associated with smokers and is much more common in males (6:1 ratio). Other risk factors include a tall, slender body type, Marfan's syndrome, pregnancy, or a positive family history. A secondary spontaneous pneumothorax may be associated with chronic lung conditions like tuberculosis, chronic obstructive pulmonary disease, cystic fibrosis and severe asthma. The incidence of spontaneous pneumothorax is between $7.4-18$ cases per 100,000 in males and $1.2-6$ cases per 100,000 in females [7]. 
A traumatic pneumothorax can be classified as non-iatrogenic or iatrogenic. Examples of causes of non-iatrogenic traumatic pneumothorax include trauma, rib fractures and high-risk sports like SCUBA diving or flying. A practitioner may induce an iatrogenic traumatic pneumothorax inadvertently when inserting a needle too far into the tissue and it punctures the pleura or lung. The incidence of acupuncture-induced pneumothorax has been reported as less than $0.01 / 10,000$ interventions [20]. This low estimate may not reflect the true frequency as many acupuncture interventions may not even include needling over the high-risk thoracic region or may become evident after a treatment and the practitioner may not be aware of it occurring.

A pneumothorax or haemothorax can present with symptoms similar to musculoskeletal pain (pain in the chest that can radiate into the shoulder or back) and will affect ventilation and impair oxygenation. These signs and symptoms will be dependent on the degree of lung collapse and may include dyspnea, increased respiratory rate, dry cough, malaise, cyanosis, and diaphoresis. They may present with decreased breath sounds on auscultation and present with a mediastinal shift. The signs and symptoms can vary as a result of a person's overall health. If in poor health, a small pneumothorax can result in severe symptoms. The rate of intrapleural gas absorption in untreated lesions is $1.25 \%$ of the thoracic volume per day. Small pneumothoraxes may reabsorb spontaneously which may result in under-diagnosing or underreporting of the $\mathrm{AE}$ [7].

The treatment of a pneumothorax is dependent on the degree of lung collapse. Patients who are clinically stable that present with a spontaneous pneumothorax of less than 3 centimeters from apex to cupola are usually treated conservatively with observation. Larger pneumothoraxes may require needle aspiration or chest tube placement [7]. Oxygen supplementation may be required if oxygen saturation levels are low.

Dry needling in the area of the upper trapezius, the thoracic erector spinae and levator scapulae region have been associated with an increased risk of iatrogenic pneumothorax. The subclavicular and supraclavicular regions, the intercostal and interspinal spaces and abnormal congenital foramen in the scapulae and sternum have also been linked with an increased risk [7]. Most iatrogenic pneumothoraxes are unilateral but cases of bilateral iatrogenic pneumothorax have been described $[7,8]$.

Knowledge and practice in the correct needling direction and needle depth, and palpation of these areas are essential when training to perform DN techniques. The lack of training, practitioner negligence, and a poor knowledge of surface and underlying anatomy can put a patient at risk of developing AE. The safe needling depth over the lungs to avoid a pneumothorax can be as small as 10-20 $\mathrm{mm}$ [7]. There are differences in the depth of the skin to the lung tissue depending on the patient's position on a intervention table [7]. Differences in the sexes and body composition (as a result of muscle thickness) can also affect the distance to the lung tissue when needling the thorax. Grusche et al. (2017) [10] reported three cases of traumatic pneumothorax following acupuncture or DN interventions seen at an emergency room in Victoria, Australia. All involved young women with low body mass indexes (BMI). Karavis et al. also presented a case study of an acupuncture-induced haemothorax involving a woman with a low BMI [7].

\section{Infections}

Infection rates with DN and acupuncture have decreased over the decades as a result of improvements in educational standards and the use of single-use disposable needles. Since 1984, the Council of Colleges of Acupuncture and Oriental Medicine have developed and administered the Clean Needle Technique (CNT) course, which is mandatory training for acupuncture licensing in the USA. These guidelines were developed to help reduce the risk of spreading infection and other risks associated with acupuncture. The CNT protocol was developed as an essential means of ensuring the safety of patients and practitioners during interventions. The course material is often updated with information from the Center for Disease Control (CDC) and the Occupational Safety and Health Administration (OSHA) as best practices change and evolve. This course is only available at present for acupuncture students but the manual, currently in the $7^{\text {th }}$ edition, is a good resource for any practitioners performing needling interventions. All physical therapists in the USA study the Occupational Safety and Health Administration's (OSHA) blood-borne pathogen regulations (standards - 29 CRF) and this will not be discussed in great detail in this paper. Detailed instruction in infection control and avoidance of needle stick injuries must be part of the dry needling training.

Infections are a concern when puncturing with a needle as the skin normally acts as a barrier to infection. Infections can be either autogenous (caused by an infectious agent that the patient already carries) or a cross-infection where a pathogen is acquired from another person or the environment. A percutaneous infection at the needle entry site will show the classic signs and symptoms of redness, swelling, local heat, and pain. Severe infections can also be accompanied by fever. Most infections related to DN are associated with mycobacterium or methicillin-resistant $\mathrm{S}$. Aureus (MRSA).

$\mathrm{Xu}$ et al. reviewed case reports published between 2000 and 2011 and found 239 cases of infection associated with acupuncture interventions [7]. The vast majority of the cases occurred in Korea, and was related to improper sterility of needles or inadequate disinfection of other equipment. It is noted that the routes of infection and their frequency have changed over the years now that disposable needles are the current clinical norm. However, the increased pervasiveness of MRSA and mycobacterium infections in healthcare settings further illustrates the continuing importance of hygiene in clinical environments. Dry needling and acupuncture can be complicated by autogenous infections caused by organisms on the patient's own skin flora. The most commonly used skin disinfectant used prior to needling is $60-70 \%$ isopropyl (or ethanol) alcohol yet some mycobacteria have been shown to be relatively resistant to it [7]. For patients with a compromised immune system, or poor hygiene, cleaning the skin with products containing $0.5 \%$ chlorhexidine or povidone iodine may be a better choice. It is noted that the formal regulations regarding skin disinfection prior to needling must be followed in a practitioner's jurisdiction [14].

Infections related to DN are rare. Hoffman reported bacterial infections caused by deep needling are uncommon because the needle tip is too small to carry a sufficient amount of inoculum from the skin [7]. Recently, however, three case studies related to DN and infections have been published. Kim et al. described a case study of a 16 year-old boy who developed an abscess in the posterolateral distal aspect of the right thigh after undergoing DN intervention for iliotibial band syndrome. The infection resolved but did require 
a hospitalization and the continuation of intravenous antibiotics for an additional three weeks after the hospital discharge. Clean needle technique was documented by the treating physical therapist and it was uncertain if the abscess development was coincidental or a clinical error associated with improper CNT [7]. Two other cases discussed the development of infections after DN with patients who had undergone previous surgical interventions. A 15 year-old who had undergone spinal arthrodesis for treatment of an idiopathic scoliosis 21 months prior developed a deep spine infection after having DN in the medial periscapular region for chronic back and shoulder pain. The periscapular abscess necessitated an operative debridement and 2 months of antibiotic therapy. The authors concluded that the deep infection from DN might have contaminated existing spinal instrumentation [7]. A Dutch case study described a 57 year-old patient who underwent a total hip replacement and then developed a bacterial infection after DN interventions 7 months post-surgery. Antibiotic interventions were unsuccessful and debridement and irrigation of the area was necessary [7]. Although there was no strong evidence for a causal relationship between DN and these infections, caution should be used in patients with joint replacements or other hardware due to the increased risk of infection.

\section{Cardiac tamponade}

Another serious, although rare, complication related to needling in the area of the sternum has been reported. The presence of a rare congenital abnormality called a sternal foramen occurs in 5-8\% of the population as a result of an incomplete fusion of the sternal plates. The foramen is usually at the level of the $4^{\text {th }}$ intercostal space but cannot be reliably palpated as tendon fibers, thin connective tissues, or bone lamella may conceal it. If a needle punctures this hole, a cardiac tamponade may occur. A compression of the heart may develop when blood or fluids build up around the pericardium, which may be fatal. The signs and symptoms of a cardiac tamponade could include chest pain, which may radiate into the neck, shoulder or abdomen, and worsens with deep breathing or coughing, anxiety, hypotension, difficulty breathing, palpitations, and fainting. Caution must be taken when needling the sternalis or pectoralis major muscle trigger points, which overlie the sternum. (It is noted that the sternalis muscle is highly variable in its presence and laterality). Injury to the pericardium or heart could occur if needling deeper than 13-25 $\mathrm{mm}$ over the sternum occurred [31]. Needling in this area must be performed in an oblique cephalad direction for safety reasons.

\section{Nerve damage (peripheral and central nervous system)}

For over a century there have been published reports of neurological damage that can occur as a result of intramuscular needling using hypodermic needles [7-9]. The resultant motor and sensory nerve damage can be catastrophic and it is noted that only $28 \%$ will have a favorable recovery [7]. The signs and symptoms associated with traumatic nerve damage will be dependent on the severity of the nerve injury. The structural and functional changes can categorize the injury as a neuropraxia, axontomesis or neurotomesis [7]. Fortunately, the smaller gauge needles used with DN interventions make complications from nerve injury far less common and serious compared with those encountered when using hypodermic needles. The most common type of nerve injury related to DN or acupuncture is a neuropraxia resulting from a hematoma causing nerve pressure. Prognosis of neuropraxia injuries is good with symptom regression generally occurring spontaneously within days or weeks. If bleeding is severe, an axontomesis could occur. Axontomesis recovery, although good, is often longer and symptoms can last for months.
Knowledge of nervous system anatomy is essential to avoid nerve injury when performing DN. Research validating needle placement in relationship to target tissues using cadavers has been done [79]. It is uncertain whether such knowledge obtained in vivo can be helpful in guiding practitioners on how to avoid other structures (like nerves) when performing DN. The use of fluoroscopy or ultrasound imaging to verify needle placement during DN is impractical [7]. Research is also continuing to determine if patient positioning during intervention can help reduce the risk of injury to nerves or other underlying tissues.

If a patient experiences a sharp electrical-type sensation distal to the needling site during $\mathrm{DN}$, a nerve may have been encountered. Peuker [31] has described several peripheral nervous system injuries associated with acupuncture and notes that peripheral nerves often have a variable anatomical course. For example, peroneal nerve palsy with a resultant foot drop development has occurred after needling the area around the fibular head. The common peroneal nerve has a variable course in $10-20 \%$ of people with a high course that is adjacent to the fibular head (near the acupuncture point location of Gallbladder 34).

More recently, McManus and Cleary described a case of a 27 yearold woman who developed a neuropraxia of her left radial nerve (at the level of the spinal groove) after DN intervention for shoulder pain. She developed a left hand muscle spasm after a needle insertion and a subsequent left wrist drop development. Despite intensive hand therapy and splinting, the patient continued to present with a profound weakness. Follow-up nerve conduction and EMG research showed no sign of nerve recovery [7].

In addition to peripheral nerve injuries, cases of injury to the spinal cord or spinal nerve roots associated with acupuncture and DN have been reported. Peuker [31] reviewed ten cases of injury that were the result of direct injury to nerves or the result of needle fragments that occurred in the cervical and lumbosacral regions. The severity of injury varied from focal neurological signs to paraplegia. The authors noted that the distance from the skin to the nerve roots or spinal cord varies from $25-45 \mathrm{~mm}$ depending on the size of the person.

\section{Needle issues - Forgotten needles or broken needles}

Practitioners must be meticulous in insuring that no needles are "forgotten" during a intervention. As most DN does not involve needle retention, this is less of a concern than acupuncture, which involves numerous needles and longer retention times. Maintaining and documenting accurate counts of needles "in" and "out" helps. Practitioners should keep used, empty needle packaging until the end of the intervention session to further help maintain a correct needle count.

Needle breakage is rare now that single-use disposable needles are the standard of practice. Prior to this, metal fatigue was a concern when needles were repetitively used and sterilized with an autoclave. The most common area for a needle to break is at the root of the needle where the shaft and the body meet. When inserting a needle, 1/4 -1/3 of the shaft should always be kept above the skin level. This requires the practitioner to use the correct needle length for the patient size and the area of the body that is being treated. Breakage may occur if there is a crack or erosion of the needle, which may be a factor when using needles of poor quality. Breakage can also occur if the patient suddenly moves or if a strong muscle spasm occurs, an external forcehits the needle, or if a practitioner tries to forcibly remove a bent needle. 
Leow et al. studied the mechanical compression forces needed to break a filiform acupuncture needle. A variety of needle diameters and lengths $(.25 \times 40 \mathrm{~mm}, .30 \times 25 \mathrm{~mm}, .18 \times 13 \mathrm{~mm})$ were bent using an Instron 3343 single column universal testing system. They were unable to break the needles but permanent bending did occur with high loads. They concluded that needle breakage is unlikely to occur with muscle spasm or sudden movement of a patient but recommended avoidance of too much force when manipulating needles [7].

If a needle does break during intervention, it is important for the patient and practitioner to stay calm and the patient to remain still. If part of the needle is visualized above the skin, it should be gently removed with forceps. If it is at the skin level, the tissue around should be gently pressed until the broken end is exposed and can then be removed. If removal is not possible, the place of needle entry on the skin should be marked and medical transportation to the hospital arranged.

Case studies have illustrated the danger of needles breaking or being "forgotten" during interventions. Chaput and Foster reported of a healthy 46-year-old woman who presented at the emergency room complaining of a foreign body sensation after eating some potato chips and feeling one lodge in her throat. An x-ray revealed a needleshaped foreign body and a subsequent laryngoscopy showed a needle in the wall of the esophagus. Six months prior she had been receiving acupuncture for a whiplash injury. She could not recall if one of the needles had broken but she had continued neck pain, which she had attributed to the motor vehicle accident [7]. Snyder also described a case of a 52 year-old man who had acupuncture treatments for chronic neck pain while out of the country on a business trip. During the session, one of the needles inserted in the upper cervical region broke. The acupuncturist was unable to remove the needle segment and the patient was transported to the hospital for $\mathrm{x}$-rays. The radiography showed that in addition to the needle near the C1-2 junction another needle was discovered at the C6-7 level, presumably from a previous acupuncture session. It was determined that both needles did not pose an immediate threat and the patient boarded a plane back to the USA. During the flight, however, the upper cervical needle migrated over 3 centimeters. Upon landing, further imaging was done and the upper cervical needle was found only two millimeters from the vertebral artery. Surgical removal of the needles was required followed by extensive physical therapy to regain cervical mobility [7].

\section{Pregnancy concerns}

Low back and pelvic girdle pain are common ailments during pregnancy. Acupuncture and DN are interventions that may be helpful in symptomatic relief of pain as pharmaceutical intervention is often not an option. It is noted that physical therapists are often reluctant to treat pregnant women with needling modalities as a defensive practice. Miscarriage occurs in over $10 \%$ of all pregnancies with $80 \%$ occurring in the first trimester. The Fanslow study showed that a random sample of 2,391 New Zealand women reported that a spontaneous abortion had affected almost 1 in 3 women [7].

Historically certain acupuncture points were considered to be "forbidden" to needle during pregnancy as they are believed to stimulate uterine contractions and, as a result, could cause a miscarriage or early labor to occur. Other acupuncture points are not needled because of potential risk of uterine penetration in the later stages of pregnancy.

It has been noted that there is considerable overlap in the locations of standard acupuncture points and myofascial trigger points $[7,8]$. Some myofascial trigger points may be in locations close to the acupuncture points that are traditionally avoided during pregnancy. For example, the area palpated for trigger points in the upper trapezius muscle is close to the acupuncture point Gallbladder 21 (Jianjing). This acupuncture point is functionally used to expedite delivery and help to promote the descent of the placenta, which is why it is not used during pregnancy. McDowell et al have summarized the correlation of the historically "forbidden" acupuncture points to myofascial trigger point locations [7].

Research into the veracity of these points as abortifacients has not been proven because of the avoidance of their use during clinical practice on pregnant subjects. An investigation into the safety of obstetric acupuncture has concluded that there is no objective evidence of harm associated by needling at the "forbidden" points after reviewing four different lines of evidence: controlled trials, observational research, animal research and a study which used acupuncture to induce miscarriage. Fifteen controlled trials (that had included one or more "forbidden" points in the study protocols) had found that there was no increase in the risk of adverse pregnancy outcomes. Observational research of 5,885 pregnant women who had acupuncture needling at "forbidden" points also showed no risk of adverse pregnancy outcomes. Acupuncture at "forbidden" points did not cause harm to healthy pregnant rats. Another study showed that the use of electro acupuncture at Spleen 6 and Large Intestine 4 acupuncture points (both considered "forbidden" during pregnancy) to terminate a mid-trimester pregnancy in 7 women was never successful. Carr concluded that acupuncture involving "forbidden" points did not increase the risk of adverse pregnancy outcome but recommended that a therapist should only treat when necessary. It is recommended that strong stimulation techniques be avoided.

Despite the lack of evidence of harm related to acupuncture or DN during pregnancy, many practitioners will avoid treating patients during the first trimester and will avoid the use of specific points. The "fear of blame" for potential miscarriage may contribute to this conservative or defense practice among physical therapists [8]. If a patient has acupuncture or DN and soon after had a spontaneous abortion, the therapist and the intervention will often be accused of causing the event [8].

\section{Conclusion}

Although there are few recorded serious AEs associated with DN, there is an absence of national or international standards in the training of physical therapists to perform $\mathrm{DN}$ interventions. In the USA there is considerable variation in DN training standards and the efficacy of the training is not regulated. The length of program is not necessarily a valid measure of competency.

The DN educational programs and federal and state professional regulatory agencies must ensure the competency of practitioners who needle these vulnerable areas. Some individual states have mandated initial competency requirements, as no profession-wide national standards exist. Individual physical therapists performing DN must always comply with the practice act standards of their own state regarding the educational and competency requirements.

At the present time, there is no consistent tracking of the AEs associated with DN. The ability to systematically report AEs associated 
Citation: Valdes VR (2019) Dry Needling in Physical Therapy Practice: Adverse Events. Int J Phys Ther Rehab 5: 157. doi: https://doi.org/10.15344/2455$7498 / 2019 / 157$

Page 8 of 9

with DN in a blame-free and voluntary way can help to focus the profession on reducing accidents [8]. Dry needling education must emphasis anatomical knowledge with advanced training when needling the trunk, thorax, head and cervical regions. It is essential that practitioners screen for individual risk factors associated with each patient, practice strict adherence to sterile needle practices and be able to recognize acute AE complications. The importance of obtaining informed consent prior to treatment, good documentation of treatment and follow up of any AEs is noted.

\section{Competing Interests}

The author declare that there is no competing interests regarding the publication of this article.

\section{References}

1. American Physical Therapy Association (APTA) Department of Practice and APTA Government Affairs (2012) Physical Therapist and the Performance of Dry Needling - An Educational Resource Paper (US) APTA

2. Caramagno J, Adrian L, Mueller L, Purl J (2015) Analysis of Competencies for Dry Needling by Physical Therapists: Final Reports.

3. Kalichman L, Vulfsons S (2010) Dry Needling in the Management of Musculoskeletal pain. J Am Board Fam Med 23: 640-646.

4. Ries E (2015) Dry Needling: Getting to the Point. Ortho PT Practice 7: 12-22.

5. Fan AY, Xu J, Li Y (2017) Evidence and Expert Opinions: Dry Needling versus Acupuncture (II)-The American Alliance for Professional Acupuncture Safety White Paper 2016. Chin J Integr Med 23: 3-9.

6. Dommerholt J, Fernandez-de-las-Penas C (2013) Trigger Point Dry Needling: An Evidence and Clinical-Based Approach. London, England: Churchill Livingstone Elsevier Ltd.

7. Dry Needling Critical Event Management. Physiotherapy alberta.

8. Edwards RI, Aronson JK (2000) Adverse drug reactions: definitions, diagnosis, and management. Lancet 356: 1255-1259.

9. Carlesso LC, MacDermid JC, Santaguida LP (2010) Standardization of Adverse Event Terminology and Reporting in Orthopaedic Physical Therapy: Application to the Cervical Spine. J Orthop Sports Phys Ther 40: 455-463.

10. Grusche F, Egerton-Warburton D (2017) Traumatic Pneumothorax Following Acupuncture: A Case Series. Clin Pract Cases Emerg Med 1: 31-32.

11. Synder DD (2019) Acupuncture gone awry: a case report of a patient who required surgical removal of two single-use filament needles following acupuncture intervention. J Manual Manip Ther 27: 180-184.

12. MacPherson $H$, Thomas $K$, Walters $S$, Fitter M, et al. (2001) A prospective survey of adverse events and intervention reactions following 34,000 consultations with professional acupuncturists. Acupunct Med 19: 93-102.

13. White A (2006) The safety of acupuncture - Evidence from the UK. Acupunct Med 24: 53-57.

14. Dommerholt J, Fernández-de-Las-Peñas C (2018) Trigger Point Dry Needling - An Evidence and Clinical-Based Approach. 2nd Edition. Elsevier Ltd.

15. Deadman P, Baker K, Al-Khafaji M (2007) A Manual of Acupuncture. Hove England. Journal of Chinese Medicine Publications.

16. Seem M (1993) A New American Acupuncture: Acupuncture Osteopathy - The Myofascial Release of the Bodymind's Holding Patterns. Denver, Colorado: Blue Poppy Press.

17. Brady S, McEvoy J, Dommerholt J, Doody C (2014) Adverse events following trigger point dry needling: a prospective survey of chartered physiotherapists. J Man Manip Ther 22: 134-140.

18. Ernst E (2010) Acupuncture - A intervention to die for? J R Soc Med 103 384-385.

19. White A (2004) A cumulative review of the range and incidence of significant adverse events associated with acupuncture. Acupunct Med 22: 122-133.
20. Witt CM, Pach D, Brinkhaus B, et al. Safety of acupuncture: results of a prospective observational study with 229,230 patients and introduction of a medical information and consent form. Forsch Komplementmed 16: 91-97.

21. Carlesso LC, Cairney J, Dolovich L, Hoogenes J (2011) Defining adverse events in manual therapy: An exploratory qualitative analysis of the patient perspective. Man Ther 16: 400-446.

22. Gilmartin S (2018) Dry Needling: A critical commentary of its effectiveness and safety profile. Physio Pract and Research 39: 155-159.

23. McDowell JM, Johnson GM (2014) Acupuncture Needling Styles and Reports of Associated Adverse Reactions to Acupuncture. Med Acupunc 26: 271-277.

24. Vulfsons S, Ratmansky M, Kalichman L (2012) Trigger Point Needling: techniques and outcome. Curr Pain Headache Rep 16: 407-412.

25. Park JE, Lee MS, Choi JY, et al. Adverse events associated with acupuncture: a prospective survey. J Altern Complement Med 16: 959-963.

26. Yamashita H, Tsukayama H, White AR, Tanno Y, Sugishita C, et al. (2001) Systematic review of adverse events following acupuncture: the Japanese literature. Compliment Ther Med 9: 98-104.

27. Furuse $N$, Shinbara $H$, Uehara A, Sugawara $M$, et al. (2017) A multicenter prospective survey of adverse events associated with acupuncture and moxibustion in Japan. Med Acupunct 29: 155-162.

28. Guidelines for safe acupuncture and dry needling practice. Australian Society of Acupuncture Physiotherapists Inc.

29. McCulloch, Michael, et al. Acupuncture Safety in Patients Receiving Anticoagulants: A Systematic Review. Perm J 19: 68-73.

30. Kouzi SA, Nuzum DS (2007) Arnica for bruising and swelling. Am J of Health Syst Pharm 64: 2434-2443.

31. Peuker E, Gronemeyer D (2001) Rare But Serious Complications of Acupuncture: Traumatic Lesions Acupunct Med 2: 103-108.

32. Berrigan WA, Whitehair CL, Zorowitz RD (2019) Acute Spinal Epidural Hematoma as a Complication of Dry Needling: A Case Report. PM R 11: 313-316.

33. Marin-Pintado-Zugasti A, Mayoral Del Moral O, Gerwin RD, et al. (2018) Post-needling soreness after myofascial trigger point dry needling: Current status and future research. J Bodyw Mov Ther 22: 941-946.

34. Travell JG, Simons DG (1999) Myofascial Pain and Dysfunction: The Trigger Point Manual Volume 1 (2nd Edition), Baltimore: Williams \& Wilkins.

35. Dommerholt J, Layton M, Hooks T, Grieve R (2015) An critical overview of the current myofascial pain literature - July 2015. J Bodyw Mov Ther 19 : 482-493.

36. Christensen KA, Gosse BJ, Hildebrand C, Gershan LA (2017) AcupunctureAssociated Vasovagal Response: Revised Terminology and Hospital Experience. Med Acupunc 29: 366-376.

37. He W, Zhao X, Li Y, Xi Q, Guo Y, et al. (2012) Adverse Events following Acupuncture: A Systematic Review of the Chinese Literature for the Years 1956-2010. J Altern Complement Med 18: 892-901.

38. Lao L, Hamilton GR, Fu J, Berman BM (2003) Is acupuncture safe? A Systematic review of case reports. Altern Ther Health Med 9: 72-83.

39. Noppen M (2010) Spontaneous pneumothorax: epidemiology, pathophysiology and cause. Eur Respir Rev 19: 217-219.

40. da Encarnação AP, Teixeira JN, Cruz JL, Oliveira JE (2014) Pneumothorax sustained during acupuncture training: a case report. Acupuncture Med 32: 514-516.

41. Patel N, Patel M, Poustinchian B (2019) Dry Needling-Induced Pneumothorax. J Am Osteopath Assoc 119: 59-62.

42. McCutcheon L, Yelland M (2011) Iatrogenic pneumothorax: safety concerns when using acupuncture or dry needling in the thoracic region. Phys The Rev 16: 126-132.

43. College of Physiotherapists of Ontario. Case of the Month: When Acupuncture Goes Very, Very Wrong.

44. Lee WM, Leung HB, Wong WC (2005) Iatrogenic bilateral pneumothorax arising from acupuncture: A case report. J Orthop Surg 13: 300-302.

45. Brett J (2017) Clean Needle Technique Manual: Best Practices for Acupuncture Needle Safety and Related Procedures. 7th Edition; USA, Council of Colleges of Acupuncture and Oriental Medicine.

46. Mitchell UH, Johnson AW, Larson RE, Seamons CT (2019) Positional changes in distance to the pleura and in muscle thickness for dry needling. Physiotherapy. 
Citation: Valdes VR (2019) Dry Needling in Physical Therapy Practice: Adverse Events. Int J Phys Ther Rehab 5: 157. doi: https://doi.org/10.15344/2455$7498 / 2019 / 157$

47. Karavis MY, Argyra E, Segredos V, Yiallouroy A, Giokas G, et al. (2015) Acupuncture-induced haemothorax: a rare iatrogenic complication of acupuncture. Acupunct Med 33: 237-241.

48. Xu S, Wang L, Cooper E, Zhang M, Manheimer E, et al. (2013) Adverse Events of Acupuncture: A Systematic Review of Case Reports. Evid Based Complement Alternat Med 2013: 581203.

49. Woo PC, Leung KW, Wong SS, Chong KT, Cheung EY, et al. (2002) Relatively alcohol-resistant mycobacteria are emerging pathogens in patients receiving acupuncture intervention. J Clin Microbiol 40: 1219-1224.

50. Hoffman $P$ (2001) Skin disinfection and acupuncture. Acupunct Med 19: 112-116.

51. Kim DC, Glenzer S, Johnson A, Nimityongskul P (2018) Deep Infection Following Dry Needling in a Young Athlete: An Underreported Complication of an Increasingly Prevalent Modality: A Case Report. JBJS Case Connect 8 : e73.

52. Callan AK, Bauer JM, Martus JE (2016) Deep Spine Infection After Acupuncture in the Setting of Spinal Instrumentation. Spine Deform 4: 156161.

53. Stenntjes K, de Vries LM, Ridwan BU, Wijgman AJ (2016) [Infection of a hip prosthesis after dry needling] Ned Tijdschr Geneeskd 160: A9364.

54. Wexburg E (1935) Neuritis und polyneuritis. In: Bumke $O$ and Foerster $O$ (eds) Handbuch der neurologie. Berlin: Springer-Verlag.

55. Villarejo FJ, Pascual AM (1993) Injection injury of the sciatic nerve (370 cases). Child's Nerv Syst 9: 229-232.

56. Kim HJ, Park SH (2014) Sciatic nerve injection injury. J Int Med Res 42: 887 897.

57. Pandian JD, Bose S, Daniel V, Singh Y, Abraham AP, et al. (2006) Nerve injuries following intramuscular injections: a clinical and neurophysiological study from Northwest India. J Peripher Nerv Syst 11: 165-171.

58. Goodman CC, Fuller KS (2009) Pathology: Implications for the Physical Therapist. Third Edition.St. Louis, MO: Saunders Elsevier.

59. Mesa-Jiménez JA, Sánchez-Gutiérrez J, de-la-Hoz-Aizpurua JL, Fernándezde-las-Peñas C (2015) Cadaveric validation of dry needle placement in the lateral pterygoid muscle. J Manipulative Physiol Ther 38: 145-150.

60. Hannah MC, Cope J, Palermo A, Smith W, Wacker V, et al. (2016) Comparison of two angles of approach for trigger point dry needling of the lumbar multifidus in human donors (cadavers). Man Ther 26: 160-164.

61. Fernández-de-Las-Peñas $C$, Mesa-Jiménez JA Paredes-Mancilla JA Koppenhaver SL, Fernández-Carnero S, et al. (2017) Cadaveric and Ultrasonographic Validation of Needling Placement in the Cervical Multifidus Muscle. J Manipulative Physiol Ther 40: 365-370.

62. Ball AM, Finnegan M, Koppenhaver S, Freres W, Dommerholt J, et al. (2019) The relative risk to the femoral nerve as a function of patient positioning: potential implications for trigger point dry needling of the iliacus muscle. $J$ Man Manip Ther 27: 162-171.

63. McManus R, Cleary M (2018) Radial nerve injury following dry needling BMJ Case Rep.

64. Leow MQH, Cao T, Wong YR, Tay SC (2017) Needle breakage in acupuncture: a biomechanical study Acupunct Med 35: 78-79.

65. Chaput JM, Foster T (2010) Pain in the Neck: The Enigmatic Presentation of an Embedded Acupuncture Needle. West J Emerg Med 11: 144-145.

66. Snyder DD (2019) Acupuncture gone awry: a case report of a patient who required surgical removal of two single-use filament needles following acupuncture intervention. J Man Manip Ther 27: 180-184.

67. Fanslow J, Silva M, Whitehead A, Robinson E (2008) Pregnancy outcomes and intimate partner violence in New Zealand. Aust NZ J Obstet Gynaceol 48: 391-397.

68. Melzack R, Stillwell DM, Fox EJ (1997) Trigger points and acupuncture points for pain: correlations and implications. Pain 3: 3-23.

69. Dorsher PT (2008) Can classical acupuncture points and trigger points be compared in the intervention of pain disorders? Birch's analysis revisited. J Altern Complement Med 14: 353-359.

70. McDowell JM, Kohut SH, Betts D (2019) Safe acupuncture and dry needling during pregnancy: New Zealand physiotherapists' opinion and practice. J Integr Med 17: 30-37.

71. Carr DJ (2015) The Safety of obstetric acupuncture: "forbidden" points revisited. Acupunct Med 33: 413-419.
72. The Physiotherapy Acupuncture Association of New Zealand (2017) Guidelines for safe acupuncture and dry needling practice. Wellington: Physiotherapy Acupuncture Association of New Zealand Inc.

73. Physiotherapy Acupuncture of Association of New Zealand (2000) Introductory manual. Dunedin: PAANZ.

74. White A (2004) Towards greater safety in acupuncture practice - a systems approach. Acupunct Med 22: 34-39.

75. Gunn CC (1996) Intervention of Chronic Pain. 2nd ed. Churchill Livingston.

76. Baldry PE (2001) Myofascial Pain and Fibromyalgia Syndromes: A Clinical Guide to Diagnosis and Management. Edinburgh, Scotland: Churchill Livingstone.

77. Fisher AA (1997) Intervention of myofascial pain. Muscoskelet Pain 7: 131142.

78. Acupuncture Canada (2015) What is Acupuncture?

79. White A (2009) Western Medical acupuncture: a definition. Acupunct Med 27: 33-35.

80. White A, Hayhoe S, Hart A, Ernst E, BMAS and AACP, et al. (2001) Survey of adverse events following acupuncture (SAFA): a prospective study of 32,000 consultations. Acupunct Med 19: 84-92.

81. Ernst E, White AR (2001) Prospective research of the safety of acupuncture: a systematic review. Am J Med 110: 481-485.

82. Melchart D, Weidenhammer W, Streng A, Reitmayr S, Hoppe A, et al. (2004) Prospective investigation of adverse effects of acupuncture in 97,733 patients. Arch Intern Med 164: 104-105.

83. Ernst E, Lee MS, Choi TY (2011) Acupuncture: Does it alleviate pain and are there serious risks? A review of reviews. Pain 152: 755-764. 